\title{
Fas receptor is required for estrogen deficiency-induced bone loss in mice
}

\author{
Natasa Kovacic ${ }^{1,2}$, Danka Grcevic ${ }^{2,3}$, Vedran Katavic ${ }^{1,2}$, Ivan Kresimir Lukic ${ }^{4}$, Vladimir Grubisic ${ }^{2}$, Karlo Mihovilovic ${ }^{2}$, \\ Hrvoje Cvija ${ }^{2,3}$, Peter lan Croucher $^{5}$ and Ana Marusic 2,6
}

Bone mass is determined by bone cell differentiation, activity, and death, which mainly occur through apoptosis. Apoptosis can be triggered by death receptor Fas (CD95), expressed on osteoblasts and osteoclasts and may be regulated by estrogen. We have previously shown that signaling through Fas inhibits osteoblast differentiation. In this study we analyzed Fas as a possible mediator of bone loss induced by estrogen withdrawal. At 4 weeks after ovariectomy (OVX), Fas gene expression was greater in osteoblasts and lower in osteoclasts in ovariectomized C57BL/6J (wild type (wt)) mice compared with sham-operated animals. OVX was unable to induce bone loss in mice with a gene knockout for Fas (Fas -/- mice). The number of osteoclasts increased in wt mice after OVX, whereas it remained unchanged in Fas -/- mice. OVX induced greater stimulation of osteoblastogenesis in Fas -/- than in wt mice, with higher expression of osteoblastspecific genes. Direct effects on bone cell differentiation and apoptosis in vivo were confirmed in vitro, in which addition of estradiol decreased Fas expression and partially abrogated the apoptotic and differentiation-inhibitory effect of Fas in osteoblast lineage cells, while having no effect on Fas-induced apoptosis in osteoclast lineage cells. In conclusion, the Fas receptor has an important role in the pathogenesis of postmenopausal osteoporosis by mediating apoptosis and inhibiting differentiation of osteoblast lineage cells. Modulation of Fas effects on bone cells may be used as a therapeutic target in the treatment of osteoresorptive disorders.

Laboratory Investigation (2010) 90, 402-413; doi:10.1038/labinvest.2009.144; published online 18 January 2010

KEYWORDS: apoptosis; CD95; knockout mice; osteoblast; osteoclast; osteoporosis

Bone mass is determined by the balance between bone formation by osteoblasts and bone resorption by osteoclasts. Among systemic factors, estrogens are major regulators of bone mass, as estrogen deficiency leads to an increase in bone resorption over bone formation and subsequent bone loss; this effect is mediated by increased osteoclastogenesis, ${ }^{1}$ as well as increased apoptosis of osteoblast lineage cells. ${ }^{2,3}$

Apoptosis can be triggered by various intra- or extracellular stimuli exerting an effect through two basic pathways: internal and external. The internal apoptotic pathway is induced by intracellular events leading to mitochondrial cytochrome $c$ release and activation of caspase $9 .^{4}$ The external apoptotic pathway is induced through death receptors of the TNF receptor family such as Fas (CD95), ${ }^{5}$ which transduce the signal to caspase 8 through the Fas-associated death domain. ${ }^{6}$ The tissue expression of Fas and its main apoptosis-inducing ligand, Fas ligand (Fasl, CD178) may be affected by estrogen. ${ }^{7-9}$

In view of the reports that human postmenopausal osteoblasts constitutively express Fas, ${ }^{10}$ and our previous finding that activation of Fas receptor directly inhibits osteoblast differentiation, with lesser effect on apoptosis, ${ }^{11}$ we hypothesized that the inhibition of differentiation and apoptosis of osteoblast lineage cells through Fas/Fasl system would mediate the effects of estrogen withdrawal on bone. To test this hypothesis we assessed how estrogen withdrawal induced by OVX affected trabecular bone volume, osteoclast, and osteoblast differentiation in mice with a gene knockout for Fas (Fas -/-). In addition, we tested in vitro whether estrogen was able to directly modulate the effect of Fas on

\footnotetext{
${ }^{1}$ Department of Anatomy, University of Zagreb, School of Medicine, Zagreb, Croatia; ${ }^{2}$ Laboratory for Molecular Immunology, University of Zagreb, School of Medicine, Zagreb, Croatia; ${ }^{3}$ Department of Physiology and Immunology, University of Zagreb, School of Medicine, Zagreb, Croatia; ${ }^{4}$ Biosistemi, Zagreb, Croatia; ${ }^{5}$ Academic Unit of Bone Biology, University of Sheffield Medical School, Sheffield, UK and ${ }^{6}$ Department of Anatomy, University of Split School of Medicine, Split, Croatia Correspondence: Dr N Kovacic, PhD, Department of Anatomy, University of Zagreb, School of Medicine, Salata 11, Zagreb, HR-10000, Croatia. E-mail: natasa@mef.hr 
osteoblasts and osteoclasts in vitro. In this study we report that the inhibition of Fas/Fasl signaling has a protective effect against estrogen withdrawal-induced osteoporosis.

\section{MATERIALS AND METHODS \\ Mice}

Female C57BL/6J mice (wild type (wt)) that were 12 weeks old, and mice deficient for the Fas gene on the C57BL/6J background (Fas $-/-^{12}$ ) were used in the experiments. Fas $-/-$ mice were a kind gift from Professor Dr Markus Simon (Max Planck Institute for Immunobiology, Freiburg, Germany). All animal protocols were approved by the ethics committee of the University of Zagreb School of Medicine (Zagreb, Croatia), and experimentation was conducted in accordance with the accepted standards of humane animal care. In vivo experiments were performed four times and animals were distributed in sham-operated $(\mathrm{SH})$ and ovariectomy (OVX) groups. The first experiment involved $16 \mathrm{wt}$ (8 SH and 8 OVX) and $15 \mathrm{Fas}-1-$ mice (7 SH and $8 \mathrm{OVX}$ ). The second experiment involved $13 \mathrm{wt}$ ( $6 \mathrm{SH}$ and 7 OVX) and $11 \mathrm{Fas}-/-$ mice (5 SH and $6 \mathrm{OVX}$ ). The third experiment involved $16 \mathrm{wt}$ ( $8 \mathrm{SH}$ and $8 \mathrm{OVX}$ ) and $13 \mathrm{Fas}-/-$ mice $(6 \mathrm{SH}$ and 7 OVX). The fourth experiment involved $25 \mathrm{wt}(12 \mathrm{SH}$ and 13 OVX) and 21 Fas $-/-$ mice (10 SH and 11 OVX).

\section{Surgical Procedure}

Mice were anesthetized with 3-bromoethanol intraperitoneally according to the manufacturer's instructions (Sigma-Aldrich, Milwaukee, MI, USA). Paravertebral lumbar areas were longitudinally incised $5-7 \mathrm{~mm}$, and the ovarian fat pad was identified. Ovaries (with oviducts) were removed through a small peritoneal incision, and postoperatively visually confirmed using light microscopy. The procedure was identical for sham operation, except that ovaries were not removed. Lethality was $<2 \%$. The animals were killed at 4 weeks after surgery. Estrogen depletion after OVX was confirmed by measuring uterine fresh weight in all animals, with a significant decrease in both mice strains compared with $\mathrm{SH}$ mice $(102.8 \pm 14.9 \mathrm{mg} v s 33.0 \pm 7.2 \mathrm{mg}$ in wt mice, $P=0.001$; and $73.7 \pm 14.4$ vs $30.6 \pm 5.6 \mathrm{mg}$ in Fas $-/-$ mice, $P=0.002$ ).

From each animal, femora were used for histomorphometry and micro-computerized tomography $(\mu \mathrm{CT})$, lumbar vertebrae were collected for $\mu \mathrm{CT}$, tibiae were used for riboxynucleic acid (RNA) isolation, and bone marrow was collected for cell cultures.

\section{Histology and Histomorphometry}

Femora were fixed in $4 \%$ paraformaldehyde for $24 \mathrm{~h}$ at $4{ }^{\circ} \mathrm{C}$, and then demineralized in $14 \%$ ethylen-diamine tetraacetic acid in 3\% formaldehyde, dehydrated in increasing ethanol concentrations and embedded in paraffin. Six $\mu \mathrm{m}$ sections were cut with a Leica SM 2000 R rotational microtome (Leica, Nussloch, Germany) and stained with Goldner's trichrome or histochemically for tartrate-resistant acid phosphatase (TRAP) activity. Histomorphometric analysis was performed under Axio Imager microscope (Carl Zeiss Microimaging, Oberkochen, Germany) equipped with a charge-coupled device (CCD) camera connected to a computer with appropriate software (OsteoMeasure, OsteoMetrics, Decatur, GA, USA).

For static histomorphometry, methaphyseal regions of Goldner's trichrome-stained femora, $0.4-1.0 \mathrm{~mm}$ distally from the epiphyseal plate, were analyzed under $\times 5$ magnification. The analyzed variables included trabecular volume (BV/TV), trabecular thickness (Tb.Th, $\mathrm{mm}$ ), trabecular number (Tb.N/mm), and trabecular separation (Tb.Sp, mm), which were automatically calculated using the OsteoMeasure software.

On TRAP-stained sections, osteoclasts were identified as multinucleated red cells placed adjacent to the bone surface. Osteoclasts were counted in the whole diaphyseal areas, excluding parts $\leqslant 1 \mathrm{~mm}$ from the epiphyseal plate. Total osteoclast number was normalized to the measured bone perimeter, and expressed as the number of osteoclasts per millimeter of bone perimeter.

For dynamic histomorphometry, mice were injected with calcein and demeclocycline, $20 \mathrm{mg} / \mathrm{kg}$ each, at a 3-day interval and killed at 2 days after the demeclocycline injection. Undecalcified femora were fixed in $70 \%$ ethanol and embedded in LR White acrylic resin (London Resin, London, UK). Nine $\mu \mathrm{m}$ sections were cut and analyzed under a fluorescent microscope (Axio Imager, Zeiss) equipped with a CCD camera connected to a computer with OsteoMeasure software, and mineral apposition rate (MAR, $\mu \mathrm{m} /$ day) was automatically calculated.

\section{Micro-Computerized Tomography}

The distal metaphyses of femora and second lumbar vertebrae were scanned using a $\mu \mathrm{CT}$ system (1172SkyScan, SkyScan, Kontich, Belgium) at $50 \mathrm{kV}$ and $200 \mu \mathrm{A}$ with a 0.5 aluminum filter using a detection pixel size of $4.3 \mu \mathrm{m}$. Images were captured every $0.7^{\circ}$ through $180^{\circ}$ (vertebrae) and every $0.7^{\circ}$ through $360^{\circ}$ (femora) rotation of the bone. The scanned images were reconstructed using the SkyScan Recon software and analyzed using SkyScan CT analysis software. Three-dimensional analysis and reconstruction of trabecular bone was performed on the bone region 1-5 $\mathrm{mm}$ distal to the growth plate. The trabecular bone compartment was delineated from the cortical bone.

The following variables were determined: trabecular bone volume fraction (BV/TV, \%), trabecular number (Tb.N/mm), trabecular thickness (Tb.Th; $\mathrm{mm}$ ), and trabecular separation (Tb.Sp; mm).

\section{Cell Culture}

Osteoblasts and osteoclasts were cultured from bone marrow as previously described. ${ }^{13}$ In brief, bone marrow was flushed out from the medullar cavity, and osteoblasts were cultured in six-well culture plates at a density of $10^{6}$ cells $/ \mathrm{ml}$ in $3 \mathrm{ml}$ of minimum essential medium- $\alpha$ ( $\alpha$-MEM) supplemented with 
$10 \%$ fetal calf serum (FCS, Invitrogen, Carlsbad, CA, USA). Osteoblast differentiation was induced from culture day 7 by the addition of $50 \mu \mathrm{g} / \mathrm{ml}$ ascorbic acid, $10^{-8} \mathrm{M}$ dexamethasone, and $8 \mathrm{mM} \beta$-glycerophosphate (Sigma-Aldrich). Osteoblast colonies were identified histochemically by the activity of alkaline phosphatase (AP), using a commercially available kit (Sigma-Aldrich). Osteoclasts were cultured in 48 -well culture plates at a density of $10^{6}$ cells $/ \mathrm{ml}$ in $0.5 \mathrm{ml}$ of $\alpha$-MEM supplemented with $10 \%$ FCS (Invitrogen). Osteoclast differentiation was stimulated by the addition of $25 \mathrm{ng} /$ $\mathrm{ml}$ recombinant murine $(\mathrm{rm})$ receptor activator of NF- $\kappa \mathrm{B}$ ligand (RANKL, gift from Amgen, Thousand Oaks, CA, USA), and $15 \mathrm{ng} / \mathrm{ml} \mathrm{rm} \mathrm{macrophage-monocyte} \mathrm{colony-sti-}$ mulating factor (M-CSF, R\&D Systems, Minneapolis, MN, USA). After 6 days of culture, the plates were stained using a commercially available TRAP assay kit (Sigma-Aldrich), and osteoclasts were identified histochemically by the activity of TRAP. Cells with three or more nuclei per cell, which were stained positively for TRAP activity, were considered osteoclasts and counted. Osteoclast differentiation was further confirmed by real-time polymerase chain reaction (PCR) for calcitonin receptor (Calcr) gene expression as a marker of mature osteoclasts. ${ }^{14}$ Osteoclasts did not differentiate in cultures without RANKL and M-CSF (not shown).

For the induction of apoptosis, $0.5 \mu \mathrm{g} / \mathrm{ml}$ of hamster antimouse Fas antibody (Jo-1, BD Pharmingen), and $5 \mu \mathrm{g} / \mathrm{ml}$ protein G (Sigma-Aldrich) were added to osteoblastogenic cultures on days 8,10 , and 13 , and to osteoclastogenic cultures on days 1 and 4 , and incubated for $16 \mathrm{~h}$ at $37^{\circ} \mathrm{C}$. Cells treated with $0.5 \mu \mathrm{g} / \mathrm{ml}$ normal hamster IgG (BD Pharmingen) and $5 \mu \mathrm{g} / \mathrm{ml}$ protein $\mathrm{G}$ were used as negative controls. AntiFas-treated osteoblastogenic cultures were also analyzed for number of osteoblast colonies on culture day 14, and the expression of osteoblast differentiation genes on the culture days 9,11 , and 14 . The proportion of apoptotic and dead cells after Fas treatment was determined by Annexin V (BD Biosciences, San Jose, CA, USA) and propidium iodide (PI) staining, performed according to the manufacturer's instructions. Data were acquired using the FACSCalibur (BD Biosciences) flow cytometer, and $2 \times 10^{4}$ events per sample were analyzed using CellQuest software (BD Biosciences).

In some experiments, $10 \mathrm{nM} / \mathrm{l}$ estradiol (Sigma-Aldrich), or a corresponding volume of ethanol (used as a solvent for estradiol) used as a negative control, was added to osteoblastogenic and osteoclastogenic cultures with each medium exchange.

\section{Gene Expression Analysis}

Total RNA was extracted from bone, fresh bone marrow, and cultured cells using TriPure reagent (Roche, Basel, Switzerland). For PCR amplification, $2 \mu \mathrm{g}$ of total RNA were converted to complementary deoxyribonucleic acid (cDNA) by reverse transcriptase (Applied Biosystems, Foster City, CA, USA). The amount of cDNA corresponding to $20 \mathrm{ng}$ of reversely transcribed RNA was amplified by real-time PCR, using specific amplimer sets designed by Primer Express software (Applied Biosystems) for $\beta$-actin (sense $5^{\prime}$-CAT TGCTGACAGGATGCAGAA-3' ${ }^{\prime}$, antisense $5^{\prime}$-GCTGATCCAC ATCTGCTGGA-3'), tumor necrosis factor receptor superfamily member 11a (Tnfrsf11a, RANK, sense 5'-GACACTGA GGAGACCACCCAA- ${ }^{\prime}$, antisense 5'-ACAACGGTCCCCTG AGGACT- $3^{\prime}$ ), Tnfsf11(RANKL, sense $5^{\prime}$-AAGGAACTGCAA CACATTGTGG- $3^{\prime}$, antisense $5^{\prime}$ GCAGCATTGATGGTGAGG TG3'), colony-stimulating factor 1 receptor (Csf1r, sense $5^{\prime}$ AGTCCACGGCTCATGCTGAT- $3^{\prime}$, antisense $5^{\prime}$-TAGCTGGA GTCTCCCTCGGA- $3^{\prime}$ ), and osteocalcin (Bglap2, OC, sense 5'-CAAGCAGGAGGGCAATAAGGT-3', antisense 5'-AGGC GGTCTTCAAGCCATACT- $3^{\prime}$ ), with SYBR Green chemistry (Applied Biosystems). Expression of Fas, runt-related transcription factor 2 (Runx2), alkaline phosphatase (Akp, AP), osteoprotegerin (Tnfrsf11b, OPG), and Calcr was analyzed using commercially available TaqMan Assays (Applied Biosystems). Real-time PCR was conducted using an ABI Prism 7000 Sequence Detection System (Applied Biosystems). Each reaction was performed in duplicate in a $25 \mu \mathrm{l}$ reaction volume. The expression of specific genes was calculated according to the standard curve of gene expression in the calibrator sample (cDNA from bone, bone marrow, osteoblastogenic, or osteoclastogenic culture), and then normalized to the expression level of the $\beta$-actin gene ('endogenous' control).

\section{Data Analysis and Interpretation}

All experiments were repeated four times, and the results from representative experiments are presented in figures. Osteoclast and osteoblast colony numbers are expressed as mean \pm s.d. of osteoclast number in six wells of a 48 -well culture plate, or number of osteoblast colonies in three wells of six-well culture plates. Differences in the histomorphometric parameters, numbers of osteoclasts, and osteoblast colonies between $\mathrm{SH}$ and OVX, B6 and Fas $-1-$ mice were analyzed using two-tailed $t$-test, and a $P$-value of $\leqslant 0.05$ was determined as statistically significant. Real-time PCR data shown in figures are expressed as mean \pm s.d. of relative messenger (m)RNA quantity in each reaction, for the representative experiment as s.d. values for real-time PCR data present variability between the technical replicates, within the single experiment. The methodological studies of reverse transcription and real-time PCR suggest that the least difference in mRNA that can be reproducibly detected is $\sim 100 \%,{ }^{15}$ and therefore we assume $100 \%$ or higher difference in gene expression, repeating through all experiments, as biologically significant. Biologically significant differences were statistically confirmed by analyzing corresponding variables from repeated experiments as independent samples $(n=4)$, using $t$-test or ANOVA.

\section{RESULTS}

\section{Ovariectomy Increases Fas Expression by Osteoblasts}

To assess the effect of estrogen withdrawal on the expression of Fas and Fasl in vivo, we analyzed their expression in the 
bone, bone marrow, and bone marrow-derived osteoblastogenic and osteoclastogenic cultures in wt mice at 4 weeks after OVX.

At 1 month after surgery, there were no significant differences in Fas or Fasl mRNA levels between OVX and
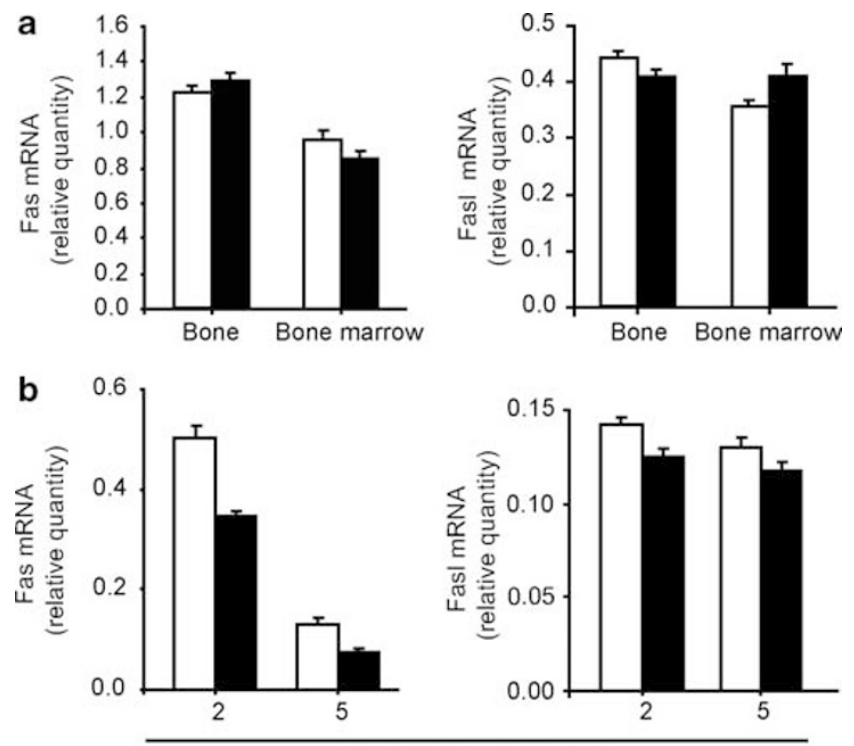

Day of osteoclast culture

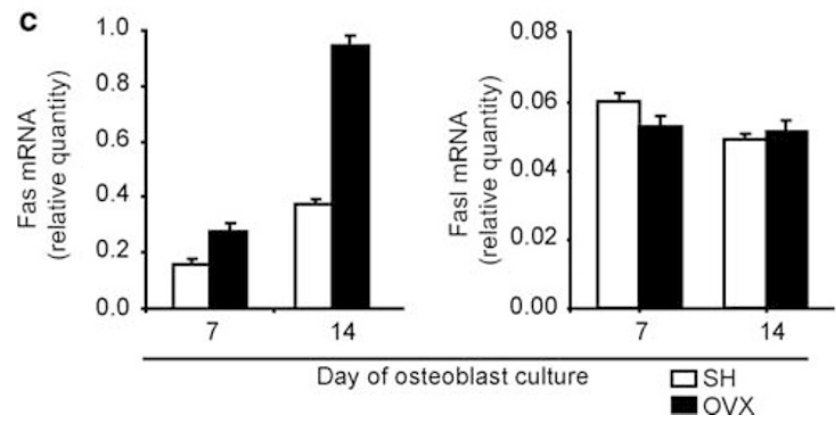

Figure 1 Gene expression of Fas and Fasl in bone, bone marrow, osteoblast, and osteoclast lineage cells in wild-type mice at 4 weeks after ovariectomy (OVX). (a) Expression of Fas/Fasl mRNA in bone and bone marrow. (b) Expression of Fas/Fasl mRNA in osteoclastogenic cultures. (c) Expression of Fas/Fasl mRNA in osteoblastogenic cultures. Expression was calculated according to the standard curve for Fas/Fasl expression in the calibrator sample (cDNA from bone, bone marrow, osteoblastogenic, or osteoblastogenic cultures), and normalized to the mRNA quantity for $\beta$-actin ('endogenous' control). Results are arithmetic mean \pm s.d. of realtime PCR reaction duplicates prepared from the same sample of the representative experiment. control mice, in either the bone or bone marrow (Figure 1a). The gene expression patterns of Fas and Fasl in osteoclastogenic cultures from bone marrow of OVX mice corresponded to the patterns observed in the cultures from SH animals, but the level of Fas expression in OVX was reduced by half compared with $\mathrm{SH}$ mice on culture day 5 (Figure 1b). For this time point, the significant difference in Fas expression was confirmed by analyzing the data obtained in four repeated experiments as independent samples $(0.16 \pm 0.02$ in $\mathrm{SH} v s 0.07 \pm 0.02$ in OVX group, $P=0.04, t$-test). Fasl gene expression was similar in SH and OVX mice during osteoclast differentiation in vitro (Figure 1b).

In four repeated experiments of osteoblastogenic culture, the level of Fas mRNA in OVX mice was almost twofold higher at day $7(0.18 \pm 0.07$ in $\mathrm{SH} v s 0.30 \pm 0.11$ in OVX group, $P=0.04, t$-test), and even more elevated at later stages of osteoblastogenesis (day 14; $0.42 \pm 0.06$ in SH vs $0.88 \pm 0.10$ in OVX group, $P=0.004, t$-test) (Figure 1c). At the same time, gene expression of Fasl in the osteoblastogenic culture was not affected by OVX (Figure 1c).

\section{Fas-Deficient Mice do not Develop Bone Loss after Estrogen Withdrawal}

After we showed the changes in the expression of Fas in bone cell cultures from wt mice at 4 weeks after OVX, we tested whether Fas was directly involved in bone loss induced by estrogen withdrawal in vivo. For this purpose we performed OVX in mice with a complete absence of Fas due to a gene knockout (Fas -/- mice) and analyzed the trabecular bone in axial (second lumbar vertebra) and appendicular (distal femur) skeleton. As expected, static histomorphometry of distal femora in OVX wt mice revealed a reduction in trabecular bone volume (BV/TV) and trabecular thickness (Tb.Th), as well as increased trabecular separation (Tb.Sp) in comparison with SH wt mice at 4 weeks after surgery (Figure 2a). In contrast, there were no statistically significant differences in trabecular bone between OVX and SH Fas $-1-$ mice at the same time point after surgery (Figure 2a).

Trabecular bone loss at all skeletal sites in OVX wt mice was confirmed using $\mu \mathrm{CT}$ analysis of distal femora and second lumbar vertebrae, whereas no changes in bone structure was observed after OVX in Fas $-/-$ mice (Figures $2 \mathrm{~b}$ and $\mathrm{c}$ ).

Figure 2 Histomorphometry and $\mu \mathrm{CT}$ analysis of femora and lumbar vertebrae from wild-type (B6) and Fas-deficient (Fas -/-) mice at 4 weeks after ovariectomy (OVX). The following histomorphometric variables were analyzed: trabecular bone volume (BV/TV), trabecular number (Tb.N/mm), trabecular thickness (Tb.Th; mm), and trabecular separation (Tb.Sp; $\mathrm{mm}$ ). (a) Upper panels, representative femoral diaphyseal sections from sham-operated (SH) and OVX B6 and Fas -/- mice, stained with Goldner-trichrome; lower panels, histomorphometric variables in SH and OVX B6 and Fas -/- mice assessed using histomorphometric analysis of distal femoral sections. ${ }^{*} P \leqslant 0.001$ ( $t$-test) vs SH mice. (b) Upper panels, representative images of distal femora from SH and OVX B6 and Fas -/- mice; lower panels, histomorphometric variables in distal femora from SH and OVX B6 and Fas -/- mice, assessed using $\mu$ CT analysis. ${ }^{*} P \leqslant 0.001$ ( $t$-test) vs SH mice. (c) Upper panels, representative images of second lumbar vertebrae from SH and OVX B6 and Fas $-/-$ mice; lower panels, histomorphometric variables in lumbar vertebrae from SH and OVX B6 and Fas $-/-$ mice, assessed using $\mu C T$ analysis. ${ }^{*} P \leqslant 0.001$ ( $t$-test) vs SH mice. Results are arithmetic mean \pm s.d. of variables measured in the single bone of each group of mice. 

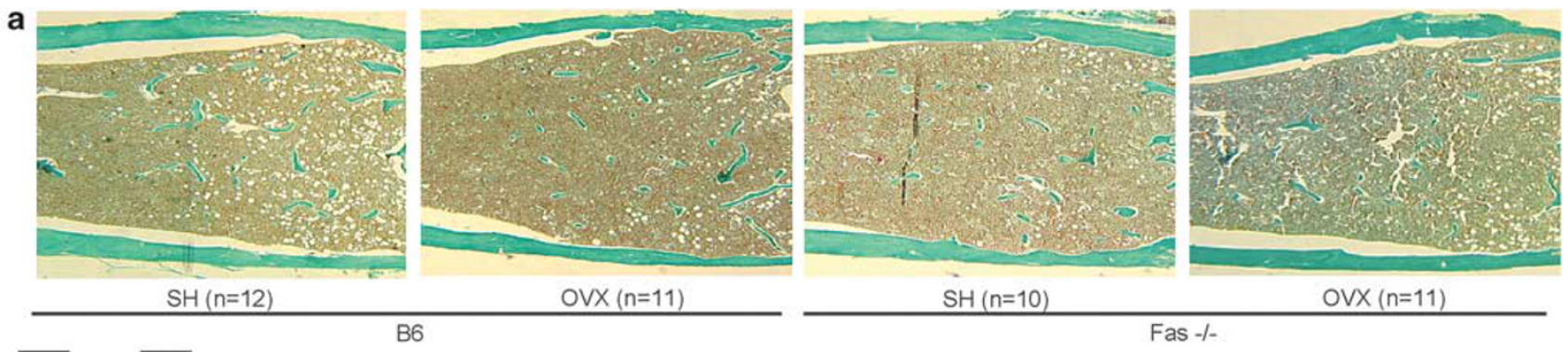

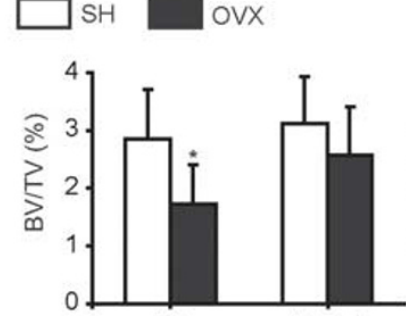

B6

b

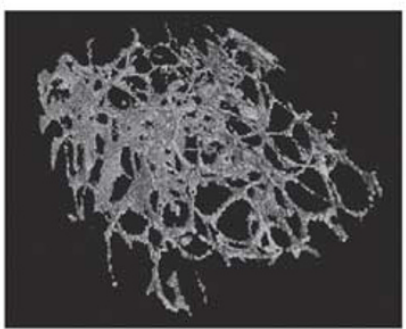

$\mathrm{SH}(\mathrm{n}=12)$

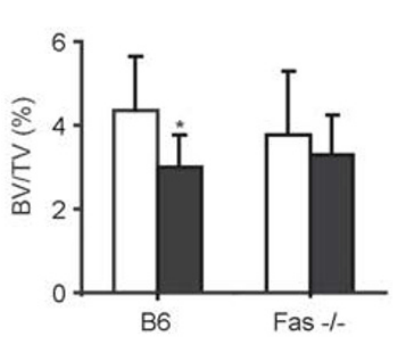

C

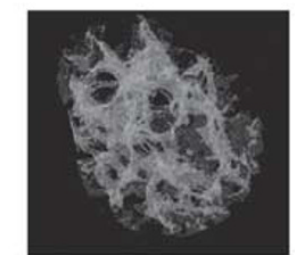

$\mathrm{SH}(\mathrm{n}=12)$

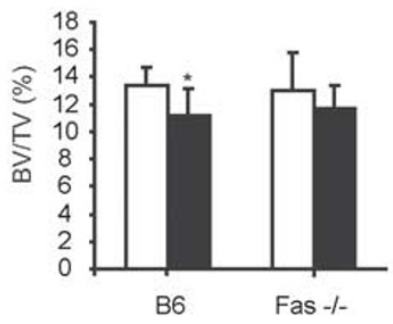

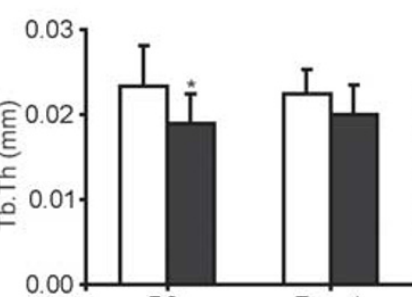

B6

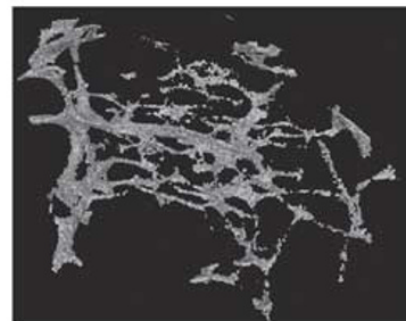

OVX (n=13)

B6

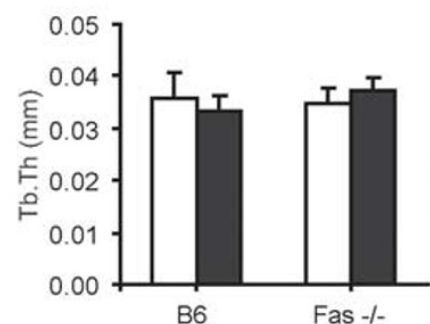

B6

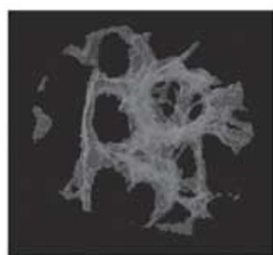

$\operatorname{OVX}(n=11)$

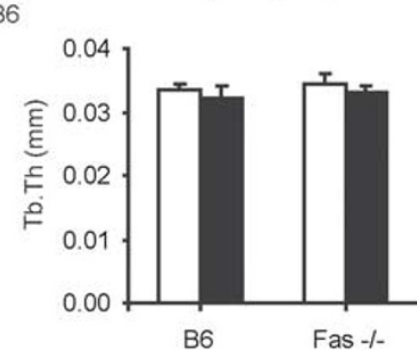

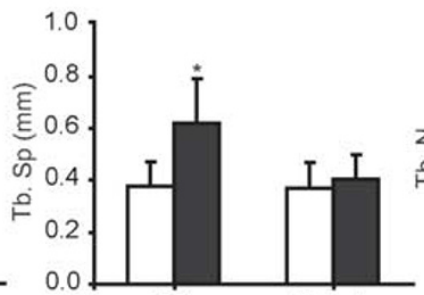

B6

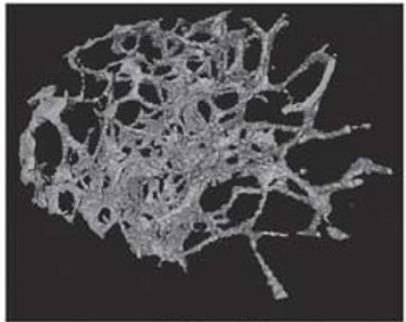

$\mathrm{SH}(\mathrm{n}=10)$
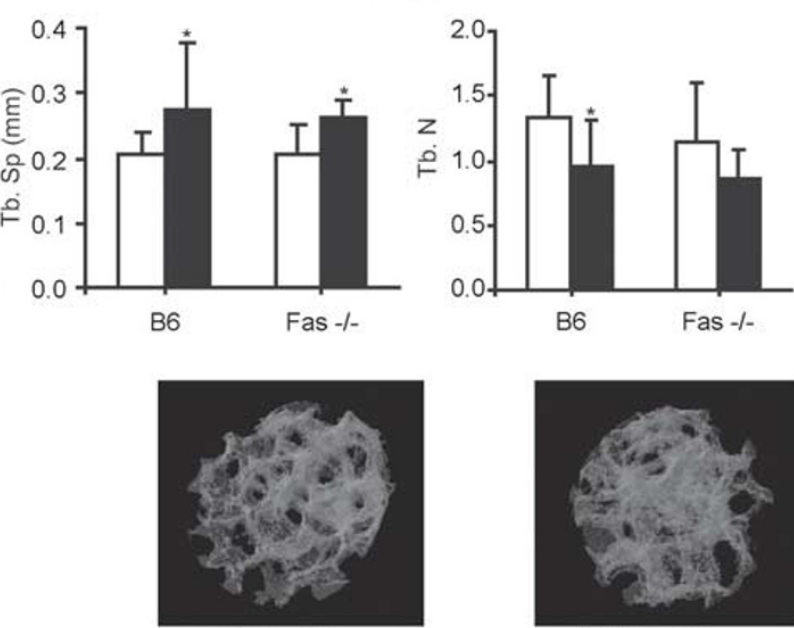

$\mathrm{SH}(\mathrm{n}=10)$

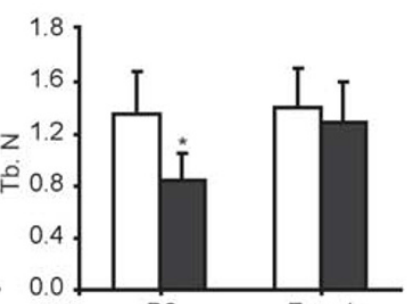

B6

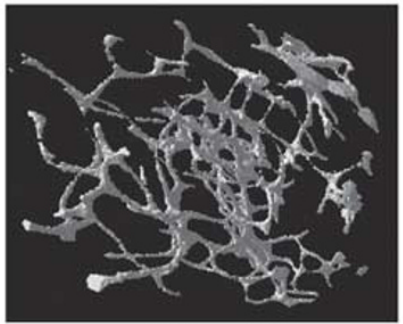

OVX (n=11)

as -1 -

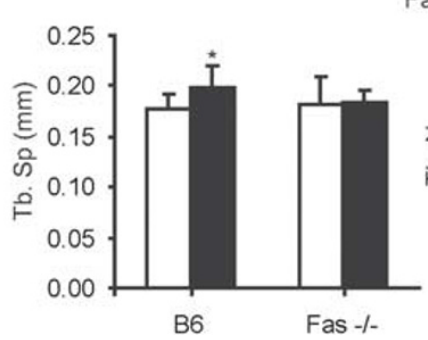

Fas -1 -

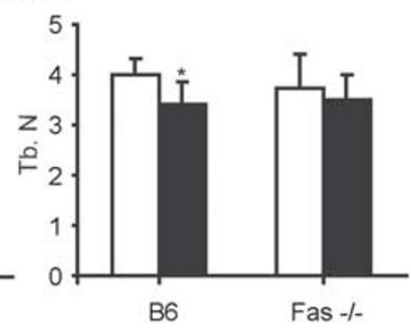

Figure 2 For caption see page 405 


\section{Estrogen Deficiency does not Stimulate Osteoclastogenesis in Fas-Deficient Mice}

As bone loss after OVX is a consequence of an increase in the number of osteoclasts, ${ }^{1}$ we analyzed whether an increase in osteoclast number could be detected in the bones of OVX Fas $-/-$ mice. Bone resorption activity on femoral sections was assessed through the number of bone-lining osteoclasts histochemically positive for TRAP activity. The number of osteoclasts per millimeter bone surface was significantly higher in OVX wt mice (compared with $\mathrm{SH}$ controls), whereas the number of osteoclasts per millimeter bone surface in OVX Fas -/- mice was unchanged, compared with SH Fas -/- mice (Figure 3a).
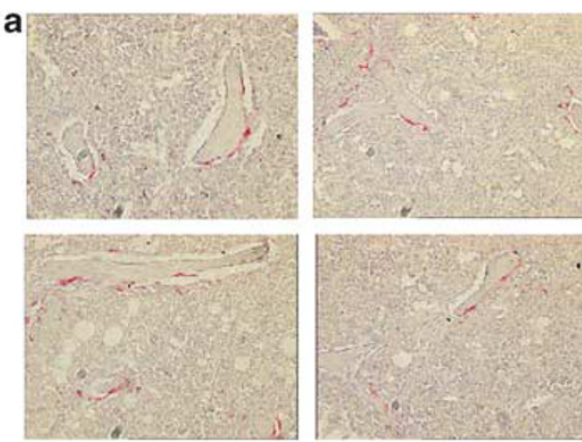

B6

b

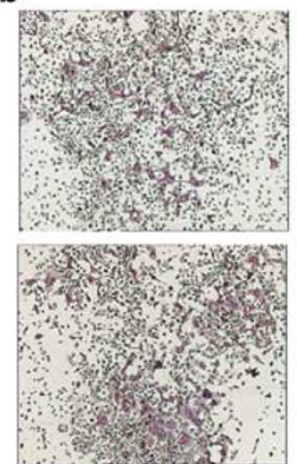

B6

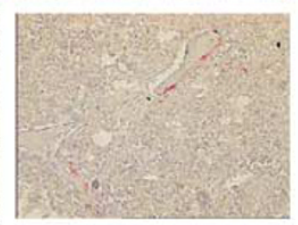

Fas $-1-$

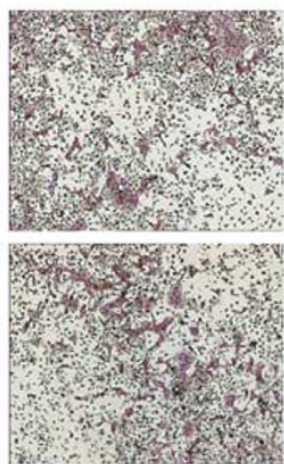

Fas -1-
$\mathrm{SH}$

ovx

$\mathrm{SH}$
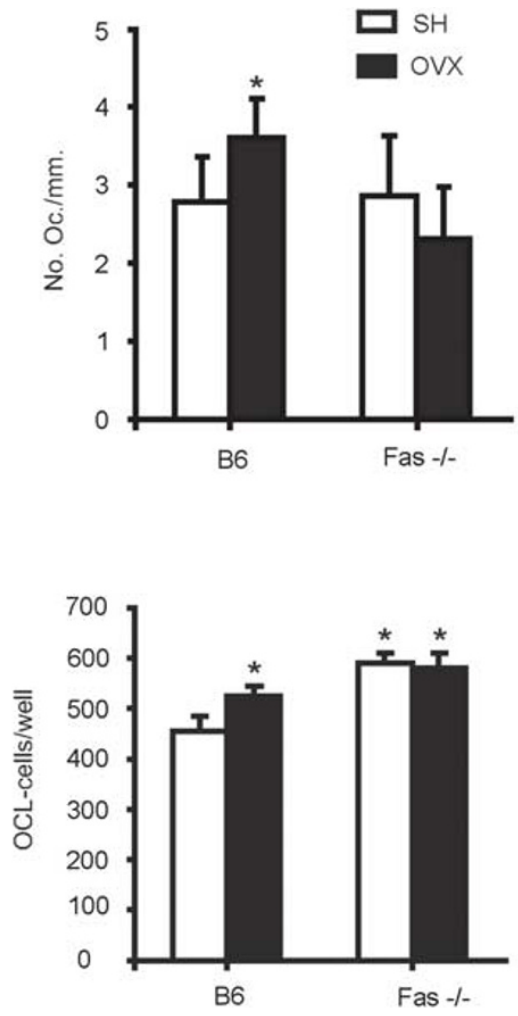

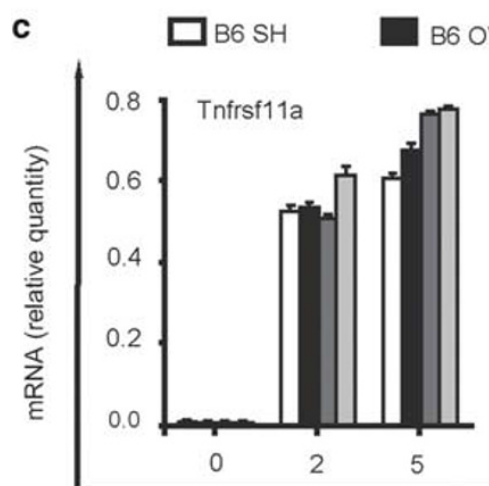

B6 ovx

Fas $-1-\mathrm{SH}$
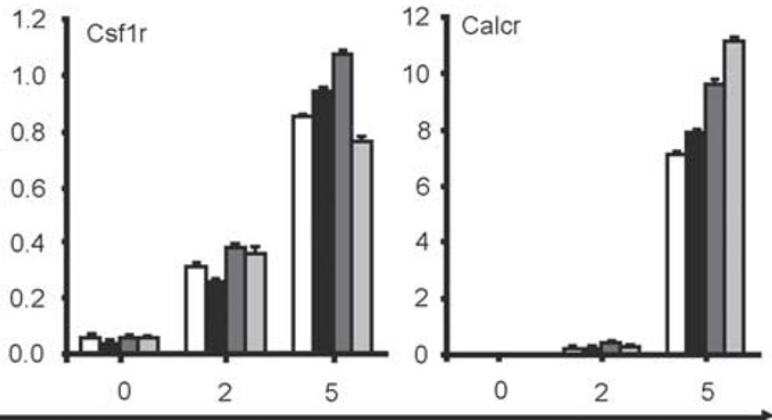

Day of osteoclast culture

Figure 3 Osteoclastogenesis in wild-type (B6) and Fas-deficient (Fas -/-) mice at 4 weeks after ovariectomy (OVX). Osteoclastogenesis in vivo was assessed using histomorphometric analysis of distal femoral sections from sham-operated (SH) and OVX B6 and Fas -/- mice stained histochemically for the activity of tartrate-resistant acid phosphatase (TRAP). Osteoclastogenesis in vitro was assessed in cultures prepared from bone marrow of SH and OVX B6 and Fas $-/$ - mice. (a) Number of TRAP-positive osteoclasts per millimeter bone perimeter in distal femoral metaphyseal sections (mean $\pm s . d$., $t$-test, ${ }^{*} P \leqslant 0.03$ vs SH mice). (b) Number of TRAP-positive osteoclasts (mean \pm s.d., $t$-test, ${ }^{*} P \leqslant 0.02$ vs SH B6 mice) on day 6 of cell culture. (c) Gene expression pattern in osteoclastogenic cultures from SH and OVX B6 and Fas -/- mice. For each time point in each group, cells were cultured in quadruplicates and pooled for RNA isolation. Day 0 represents gene expression in freshly isolated bone marrow cells. Values were calculated according to the standard curve of gene expression in the calibrator sample (cDNA from osteoclastogenic cultures) and normalized to the expression of the gene for $\beta$-actin ('endogenous' control). Results are arithmetic mean \pm s.d. of real-time PCR reaction duplicates prepared from the same sample of the representative experiment. Csf1r, colonystimulating factor 1 receptor; Calcr, calcitonin receptor; Tnfrsf11a, tumor necrosis factor receptor superfamily member $11 a$, RANK. 
Although SH Fas -/- mice had a more robust osteoclastogenesis from the bone marrow progenitors in vitro compared with wt mice (590.0 \pm 20.0 per well, $v s 457.5 \pm 26.3$ per well in wt mice, $t$-test, $P \leqslant 0.001$ ), the number of osteoclasts differentiated from Fas -/- bone marrow remained unchanged after OVX (Figure 3b). To assess the osteoclast

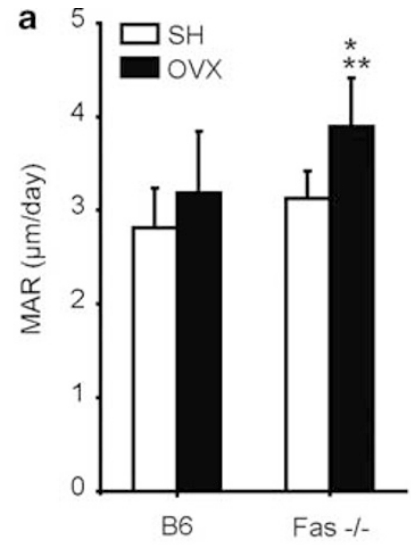

b

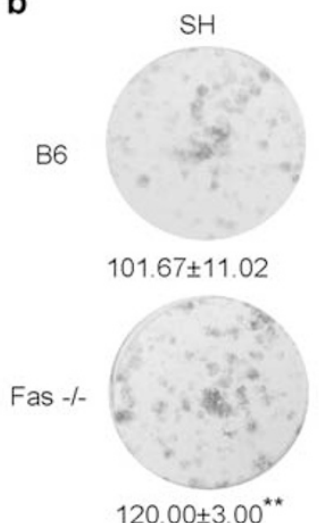

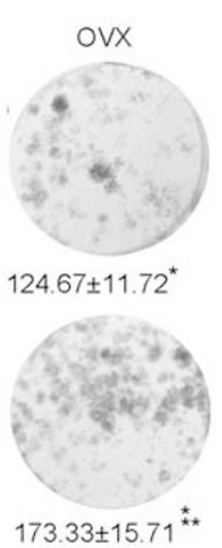

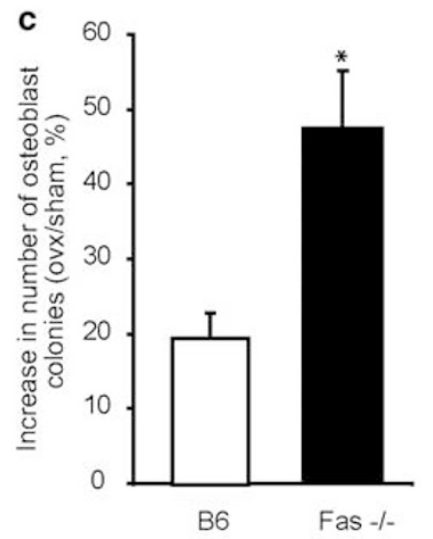

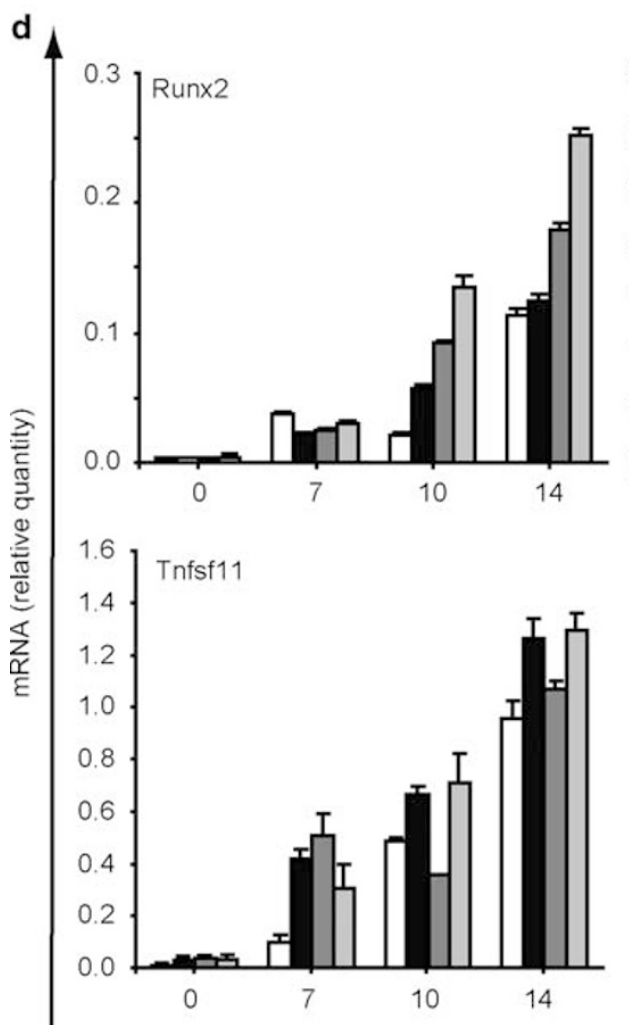
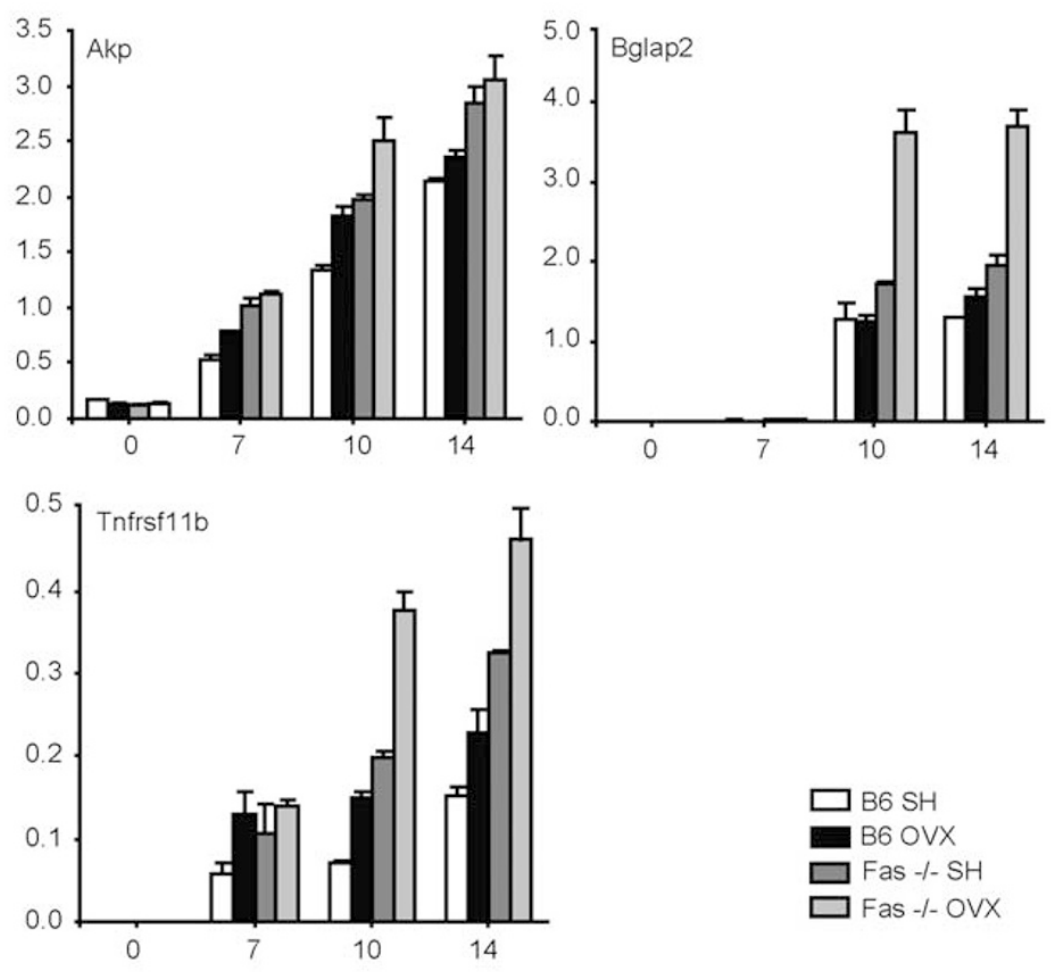

Day of osteoblast culture

Figure 4 Osteoblast differentiation and activity in wild-type (B6) and Fas-deficient (Fas -/-) mice at 4 weeks after ovariectomy (OVX). Osteoblast activity in vivo was assessed using dynamic histomorphometry. Osteoblastogenic cultures were prepared from bone marrow of sham-operated (SH) and OVX B6 and Fas -/- mice. (a) Mineral apposition rate (MAR) in SH and OVX B6 and Fas -/- mice was assessed using histomorphometric analysis of distal femoral sections: ${ }^{\star} P \leqslant 0.001$ (t-test) vs SH mice, ${ }^{* * P} \leqslant 0.001$ (t-test) vs B6 mice. (b) Osteoblastic colonies from SH and OVX B6 and Fas $-/-$ mice on day 14 of cell culture, stained red for alkaline phosphatase (AP). Number of osteoblast colonies is presented as mean \pm s.d. of culture duplicates: $t$-test, ${ }^{\star} P \leqslant 0.05$ vs SH mice, ${ }^{* * P} \leqslant 0.05$ vs B6 mice (c) Percentage increase in the number of osteoblast colonies in OVX mice compared with SH mice (mean \pm s.d. increase in four repeated experiments; $t$-test, ${ }^{\star} P=0.002$ vs B6 mice). (d) Gene expression pattern during in vitro osteoblast differentiation. For each time point in each group, cells were cultured in triplicates pooled for RNA isolation. Day 0 represents gene expression in freshly isolated bone marrow cells. Values were calculated according to the standard curve of gene expression in the calibrator sample (cDNA from osteoblastogenic culture), and normalized to the expression of the gene for $\beta$-actin ('endogenous' control). Results are arithmetic mean \pm s.d. of real-time PCR reaction duplicates prepared from the same sample of the representative experiment. Akp, alkaline phosphatase gene; AP, alkaline phosphatase; Bglap2, osteocalcin; Runx2, runt-related transcription factor 2; Tnfsf11, tumor necrosis factor receptor superfamily member 11, RANKL; Tnfrsf11b, tumor necrosis factor receptor superfamily member $11 \mathrm{~b}$, OPG. The color reproduction of this figure is available on the html full text version of the manuscript. 
differentiation sequence from bone marrow cells, we followed the dynamics of expression of three key osteoclast-related genes: Tnfrsfl1a (RANK), Csf1r, and Calcr. As shown in Figure $3 c$, the expression pattern of these genes was comparable in all four groups of animals, indicating that the differentiation sequence was not affected by the OVX in both wt and Fas $-/-$ mice.

\section{Estrogen Deficiency Stimulates Osteoblast Activity and Differentiation in Fas-Deficient Mice}

MAR was used to estimate osteoblast activity in vivo. Although MAR increased after OVX in wt and Fas -/- mice, this increase was statistically significant only in Fas $-/-$ mice (Figure 4a).

Osteoblastogenesis in vitro was assessed by the number of formed osteoblast colonies histochemically positive for AP activity and by determining the expression of osteoblast differentiation genes: Runx2, Akp, Tnfsf11 (RANKL), Tnfrsf11b (OPG), and Bglap2 (OC). The number of osteoblast colonies was higher in Fas $-/-$ mice, either SH or OVX, in comparison with the wt animals (Figure $4 \mathrm{~b}$ ). Bone marrow from both wt and Fas -/- mice had more AP-positive colonies at 4 weeks after OVX (Figure $4 \mathrm{~b}$ ), but this increase in Fas -/- mice was twice greater than that in wt mice $(19.8 \pm 3.7 \%$ increase in wt mice $v s \quad 47.5 \pm 7.6 \%$ in Fas - /mice, $P=0.002, t$-test, Figure $4 \mathrm{c}$ ).

OVX led to an increase in the expression of osteoblast differentiation genes in both wt and Fas -/- mice; this increase being significant in Fas -/- mice especially for OPG and $\mathrm{OC}$ at days 10 and 14 of osteoblastogenic cultures $(P<0.05$, ANOVA and Student-Newman-Keuls post hoc test, Figure $4 \mathrm{~d}$ ). The expression pattern of osteoblast-differentiation genes confirmed enhanced osteoblastogenesis in Fas -/mice, and this enhancement was significant for Runx2 at later stages (days 10 and 14) of osteoblast differentiation $(P<0.05$, ANOVA and Student-Newman-Keuls post hoc test, Figure $4 d$ ). In addition, the expression of RANKL was similar in all groups of mice, giving the significant 3.5-fold decrease in RANKL/OPG ratio in Fas -/- mice, compared with wt mice on culture day 14 ( $P=0.01$, ANOVA and Student-NewmanKeuls post hoc test).

\section{Estrogen Decreases Expression of Fas and Abrogates In Vitro Effect of Fas Activation in Osteoblast Lineage Cells} As we found that the absence of Fas in vivo had a protective effect on bone loss after OVX and resulted in a stimulation of the osteoblastogenic response to estrogen withdrawal, we then assessed whether estrogen could directly modulate the effects of Fas activation on osteoblast lineage cells.

We first analyzed the effect of exogenous estradiol on Fas gene expression in osteoblast lineage cells, and showed that it negatively regulates the Fas gene expression in both immature (day 7, 70\% of the level in the control cultures) and mature (day 14, 60\% of the level in the control cultures) osteoblastogenic cultures (Figure 5a). We then assessed whether estradiol could rescue differentiating osteoblasts from Fas-induced apoptosis. As shown in Figure 5b, treatment with estradiol $24 \mathrm{~h}$ before Fas activation by Jo- 1 antibody reduced the proportion of apoptotic and dead cells by $7.5 \%$ in immature osteoblasts (culture day 11), as measured using Annexin V/PI labeling. That effect was most pronounced in mature osteoblasts (culture day 14), in which anti-Fas treatment doubled the proportion of dead and apoptotic cells, and estradiol pretreatment reduced that number to the level of the control cells (Figure 5b).

Treatment with an activating anti-Fas antibody resulted in a decrease in AP-positive osteoblast colonies on culture day 14 , which is in accordance with our previous study. ${ }^{11}$ This was not only a result of increased apoptosis, but also of a direct effect on osteoblast differentiation. Estradiol treatment before the addition of anti-Fas antibody was able to partially abrogate the inhibitory effect of Fas activation and increased the number of AP-positive colonies compared with the cultures treated with anti-Fas only (Figure 5c). Expression of Runx2 decreased in immature (days 7 and 10) osteoblastogenic cultures treated with anti-Fas antibody, and that the effect of Fas activation could be blocked by estradiol pretreatment, but only when added early in the culture course (Figure 5d). In contrast, downregulation of Akp, Tnfrsf11b, and Tnfsfl1 genes by anti-Fas treatment could not be abrogated with estradiol pretreatment (Figure 5d).

\section{Estrogen has no Effect on Fas-Induced Apoptosis of Immature Osteoclast Lineage Cells}

As our earlier study showed that Fas activation doubled the number of apoptotic cells in early stages of osteoclastogenic cultures and did not affect differentiation of osteoclasts, ${ }^{11}$ we analyzed the effect of estradiol on the expression of Fas and Fas-mediated apoptosis in osteoclastogenic cultures. We found no significant changes in the expression of Fas in osteoclastogenic cultures treated with estradiol compared with control cultures (Figure 6a). Anti-Fas antibody doubled the number of apoptotic cells on day 2 of osteoclastogenic cultures, whereas it had no effect on the number of apoptotic cells in mature osteoclastogenic cultures (day 5, Figure 6b). Estradiol pretreatment had no effect on the number of apoptotic or dead cells on day 2 of osteoclastogenic cultures, but it doubled the number of dead cells in mature osteoclastogenic cultures (day 5, Figure 6b).

\section{DISCUSSION}

The results of our study clearly showed that the effects of estrogen withdrawal were mediated by Fas. Estrogen withdrawal stimulated Fas expression in cultured osteoblasts. Fas has already been shown to be expressed on mature osteoblasts, ${ }^{11,16-18}$ in which it induces apoptosis and suppresses differentiation. ${ }^{11}$ It has been further shown that Fas is constitutively expressed in osteoblasts cultured from bones of postmenopausal women, and its activation is able to induce their apoptosis. ${ }^{10}$ Increased Fas expression on osteoblast 
lineage cells observed in our study after OVX may thus represent an effective mechanism for limiting osteoblast activity and contributing to the development of the osteoporotic phenotype.
On the other hand, we found a moderate decrease in Fas expression in osteoclastogenic cultures, which may have explained, at least in part, the expansion of the progenitor pool and increased in vitro osteoclastogenesis in response to es- a

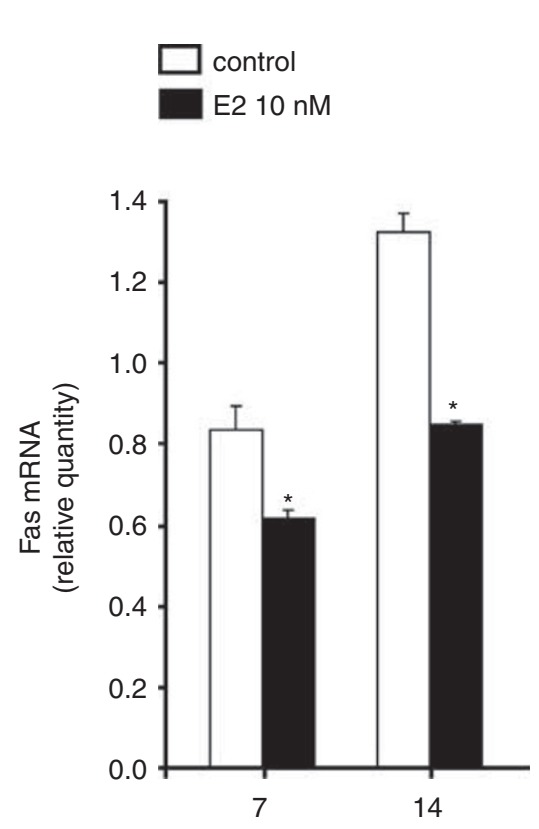

Day of osteoblast culture
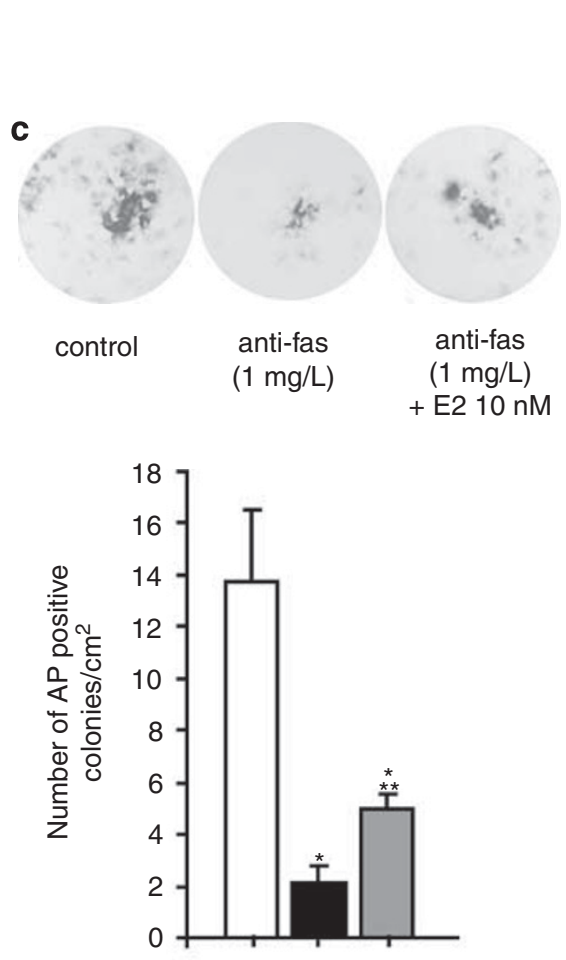
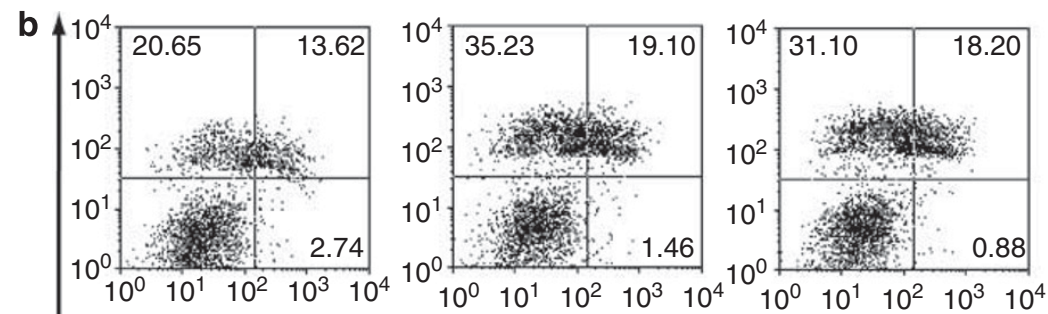

day 9
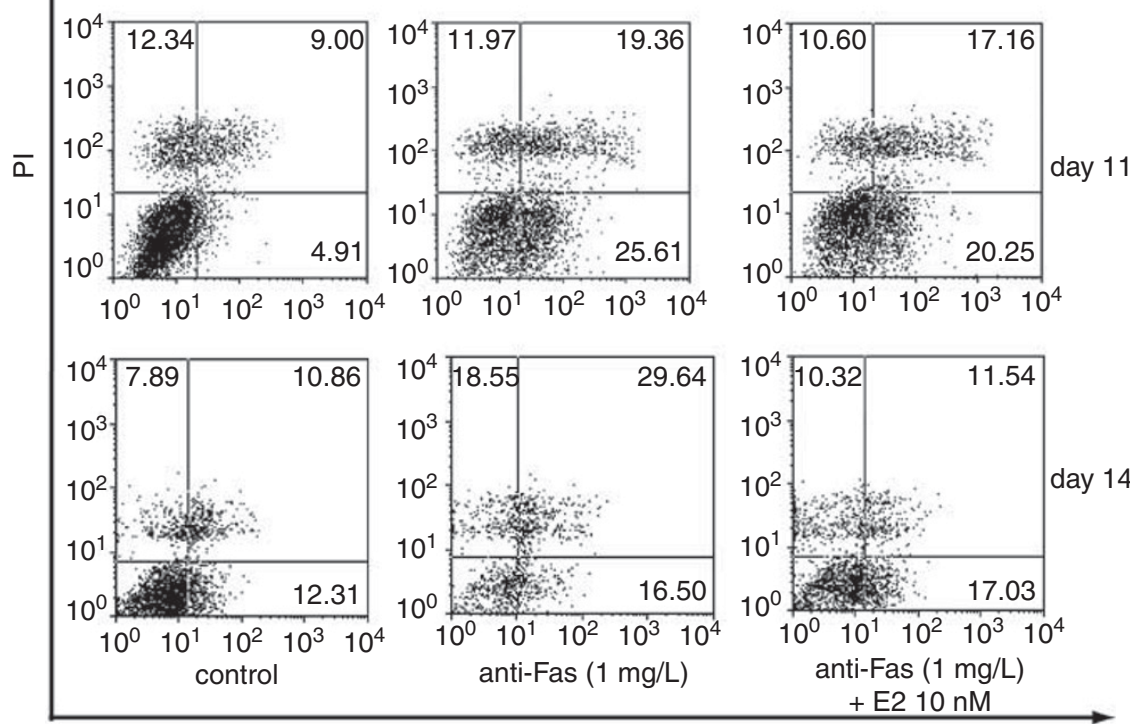

d

Annexin V - FITC
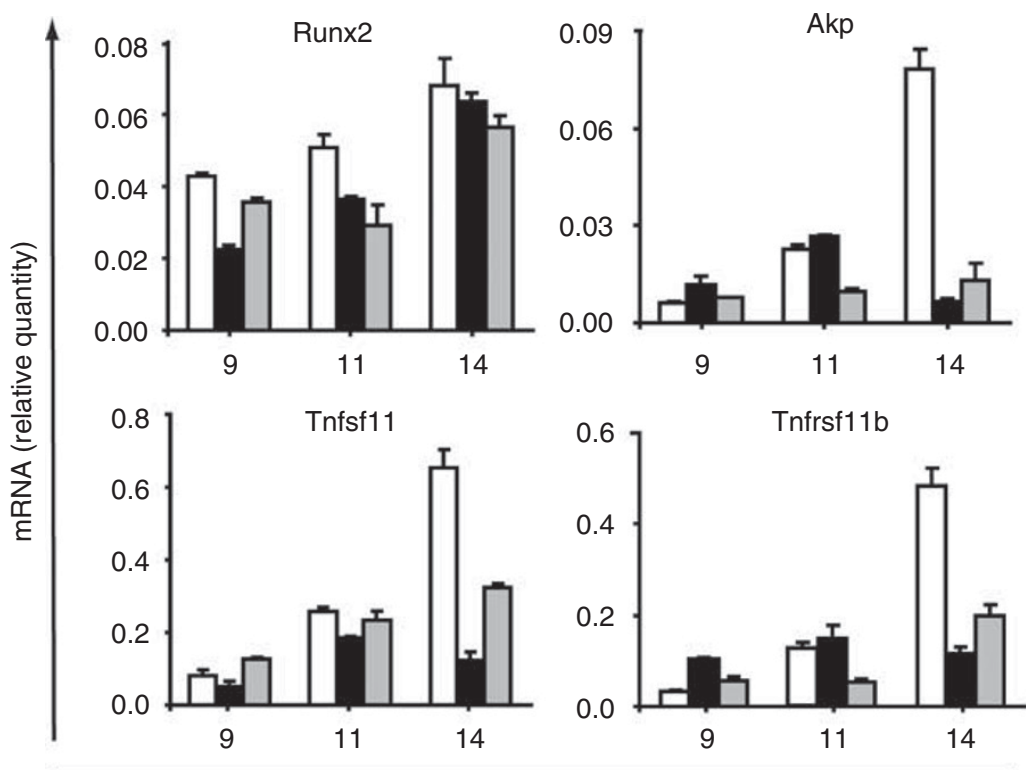

Day of osteoblast culture 
trogen withdrawal. Fas expression on osteoclast lineage cells has been described, but the data are conflicting and dependent on culture conditions. They vary from absent ${ }^{19}$ to strongly expressed and efficient in inducing apoptosis of mature osteoclasts. ${ }^{20}$ In our model, the expression of Fas mRNA on osteoclasts lineage cells was basally low (between $2^{20}$ - and $2^{19}$-fold lower than that of $\beta$-actin) and Fasl had a limited effect of inducing apoptosis in a small proportion of cells in immature osteoclastogenic cultures. ${ }^{11}$ Therefore, the absence of Fas-mediated osteoclast apoptosis may not be an important mechanism for OVX-induced increase in osteoclastogenesis.

Changes in Fas expression seem to be the main regulatory effect of estrogen withdrawal in bone cells, as expression of Fasl in bone cells was unchanged by OVX. However, Fasl is expressed in other cells present within the bone marrow microenvironment, such as activated T lymphocytes, ${ }^{21} \mathrm{NK}$ cells, ${ }^{22}$ monocytes ${ }^{23}$ and erythroid lineage cells, ${ }^{24}$ which may provide sufficient levels of endogenous Fasl to induce apoptosis and inhibit differentiation of osteoblasts.

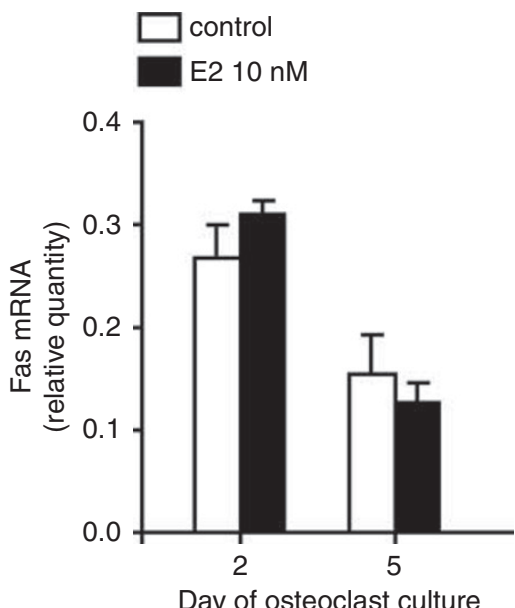

Day of osteoclast culture
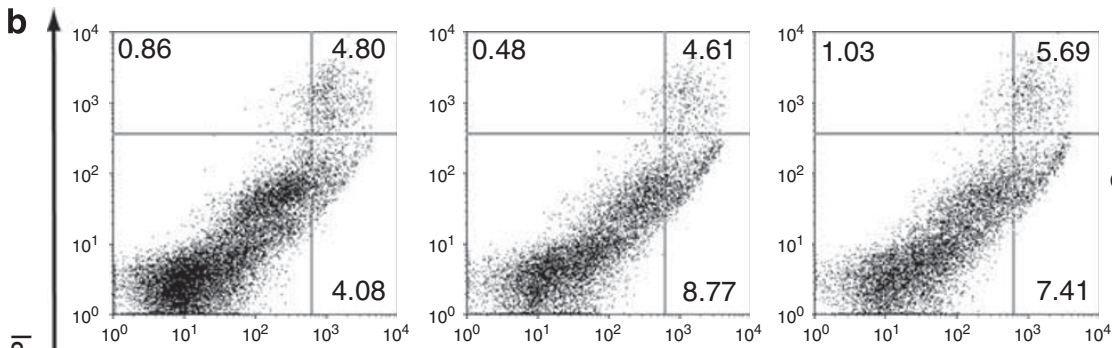

day 2

$\bar{\alpha}$
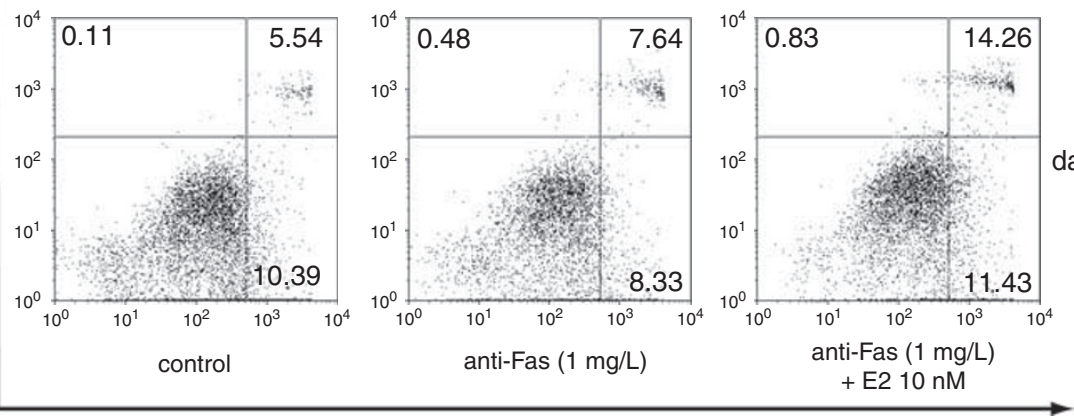

Annexin V - FITC

Figure 6 Estradiol does not influence Fas-mediated osteoclast apoptosis. Osteoclastogenic cultures were prepared from bone marrow of wt mice and treated with $0.5 \mu \mathrm{g} / \mathrm{ml}$ of anti-Fas antibody (Jo-1) and $5 \mu \mathrm{g} / \mathrm{ml}$ protein G at days 1 and 4 . Cells treated with $0.5 \mu \mathrm{g} / \mathrm{ml}$ normal hamster lgG and $5 \mu \mathrm{g} / \mathrm{ml}$ protein $\mathrm{G}$ were used as negative controls. A total of $10 \mathrm{nM}$ estradiol was added to osteoclastogenic cultures with each medium exchange and a corresponding volume of ethanol (used as a solvent for estradiol) was used as a negative control. (a) Expression of Fas in osteoclastogenic cultures after treatment with estradiol. Values were calculated according to the standard curve of gene expression in the calibrator sample (cDNA from osteoclastogenic cultures), and normalized to the expression of the gene for $\beta$-actin ('endogenous' control). Cell treatment was performed in six wells and three wells were pooled for RNA isolation. Results are arithmetic mean \pm s.d. of relative Fas mRNA expression in two pooled samples. (b) Representative data of the Annexin V-FITC/PI labeling of apoptotic cells. Numbers represent the percentages of cells in each quadrant. Apoptotic cells are in the lower right quadrant, and dead cells are in the upper right quadrant.

Figure 5 Estradiol modifies the effect of Fas on osteoblast differentiation and apoptosis. Osteoblastogenic cultures were prepared from the bone marrow of wt mice. Osteoblastogenic cultures were treated with $0.5 \mu \mathrm{g} / \mathrm{ml}$ of anti-Fas antibody (Jo-1) and $5 \mu \mathrm{g} / \mathrm{ml}$ protein G at days 8,10 , and 13 . Cells treated with $0.5 \mu \mathrm{g} / \mathrm{ml}$ normal hamster $\mathrm{lgG}$ and $5 \mu \mathrm{g} / \mathrm{ml}$ protein $\mathrm{G}$ were used as negative controls. A total of $10 \mathrm{nM}$ estradiol was added to osteoblastogenic cultures with each medium exchange and a corresponding volume of ethanol (which was used as a solvent for estradiol) was used as a negative control. (a) Expression of Fas mRNA in osteoblastogenic cultures after treatment with estradiol. Values were calculated according to the standard curve of gene expression in the calibrator sample (cDNA from osteoblastogenic cultures) and normalized to the expression of the gene for $\beta$-actin ('endogenous' control). Cell treatment was performed in triplicate wells and results are arithmetic mean \pm s.d. of relative Fas mRNA expression in each well ( ${ }^{*} P<0.05 v s$ control group, $t$-test). (b) Representative data of the Annexin V-FITC/PI labeling of apoptotic cells. Numbers represent the percentages of cells in each quadrant. Apoptotic cells are in the lower right quadrant, and dead cells are in the upper right quadrant. (c) Effect of estradiol on the number of osteoblast colonies after treatment with anti-Fas antibody. Histograms represent the number of osteoblast colonies per square centimeter plate surface (mean \pm s.d., $n=3$; ${ }^{\star} P<0.05$ vs control group, ${ }^{* * P}<0.05$ vs anti-Fas and control group, $t$-test). Figures show representative osteoblast colonies on day 14 of cell culture, stained red for AP. (d) Gene expression pattern during in vitro osteoblastic differentiation. Values were calculated according to the standard curve of gene expression in the calibrator sample (cDNA from osteoblastogenic culture) and normalized to the expression of the gene for $\beta$-actin ('endogenous' control). Results are arithmetic mean \pm s.d. of real-time PCR reaction duplicates prepared from the same sample of the representative experiment. Akp, alkaline phosphatase gene; AP, alkaline phosphatase; Runx2, runt-related transcription factor 2; Tnfsf11, tumor necrosis factor receptor superfamily member 11, RANKL; Tnfrsf11b, tumor necrosis factor receptor superfamily member 11, OPG. The color reproduction of this figure is available on the html full text version of the manuscript. 
To confirm the physiological importance of changes in Fas expression after OVX, we performed OVX in Fas -/- mice and showed that the absence of Fas would protect them from the estrogen withdrawal-induced bone loss. OVX significantly reduced the trabecular bone volume in wt mice, whereas it was unable to induce significant bone loss in Fas $-/-$ mice. These findings strongly suggest that Fas is required for bone loss after OVX in vivo, and are in line with our previous finding that mice with a point mutation for Fasl (gld) also do not lose bone after OVX. ${ }^{13}$

Fas deficiency influenced both osteoclastic and osteoblastic responses to estrogen withdrawal. OVX increased the number of osteoclasts in vivo as well as osteoclastogenesis in vitro in wt mice, whereas such an increase was not observed in Fas - I- mice. Several studies already showed the importance of Fas/Fasl system in the apoptosis of mature osteoclasts or osteoclast progenitors in the pathogenesis of osteoresorptive disorders. ${ }^{13,20,25,26}$ Increased osteoclastogenesis is a welldocumented response to estrogen deficiency, ${ }^{1}$ and there are many mechanisms proposed to be involved in this response, such as IL-6, ${ }^{1}$ absence of a direct inhibitory effect of estrogen on mature osteoclast apoptosis, ${ }^{27}$ and stimulation of osteoclastogenesis by increased TNF production from lymphocytes ${ }^{28}$ among others. As Fas was found to be expressed on osteoclasts, apoptosis induced by Fasl may be an effective mechanism limiting the osteoclast progenitor pool. In addition, a decrease in Fas expression on osteoclasts after OVX may contribute to an increased osteoclastogenesis. However, in vivo absence of Fas resulted in failure of a normal osteoclastogenic response to estrogen withdrawal. One of the potential mechanisms for this osteoprotective effect is an altered pattern of osteoblast differentiation in Fas deficiency. We have previously described increased osteoblast differentiation as a result of Fas or Fasl deficiency. ${ }^{11,13}$ Such increased differentiation is characterized by increase in the expression of $\mathrm{OPG},{ }^{11,13}$ which is not followed by an increase in the expression of RANKL, suggesting that the altered differentiation pattern of osteoblasts in Fas-deficient mice results in a lower RANKL/OPG ratio compared with wt mice, which may negatively regulate osteoclastogenesis. Furthermore, Park et $a l^{29}$ reported enhanced osteoclastogenesis in response to Fasl in vitro, but as a consequence of increased levels of TNF and IL-1 in osteoclast cultures due to Fas activation. Although the significance of this mechanism in vivo is unknown, it may at least partially contribute to the absence of an osteoclastogenic response to ovarietomy in Fas -/- mice.

Increased osteoblastogenesis, with the highest increase between weeks 2 and 8 after OVX, is also a well-documented response to OVX and has been ascribed to stimuli that are additional to factors released from the bone matrix during osteoclastic resorption, as occurs during normal remodeling. ${ }^{30}$ In our model, the increase in osteoblastogenesis was more prominent in Fas-deficient than in wt mice, suggesting involvement of Fas in limiting the osteoblastic response after OVX, and thus contributing to bone loss. Fas has also been shown to be expressed by human osteoblasts and is necessary for their apoptosis. ${ }^{31}$ TNF and IL-1 may increase the sensitivity of human osteoblasts to Fas-induced apoptosis, ${ }^{32}$ but estradiol or raloxifene could not abrogate the effect of TNF in stimulating the expression of Fas and induction of postmenopausal human osteoblast apoptosis. ${ }^{10}$ This process could be in vitro successfully inhibited by the antioxidant molecules, fraxetin and myrecitin. ${ }^{33,34}$ As increased TNF production is a well-described consequence of estrogen deficiency, ${ }^{28}$ such small molecules may be potentially used as antiresorptive agents.

The osteoprotective effect of Fas absence may be explained by a direct influence of estrogen on Fas expression and activity on bone cells, or by indirect mechanisms involving other cells and molecules that are able to influence bone cell differentiation, activity, and death.

To distinguish which of our findings resulted from direct interaction of estrogen and Fas in bone cells, we analyzed whether estrogen is able to modify the expression of Fas in osteoblastogenic and osteoclastogenic cultures. Estradiol was able to reduce the expression of Fas in immature and mature osteoblastogenic cultures, and pretreatment with estradiol before Fas stimulation resulted in fewer dead cells in mature osteoblastogenic cultures, while having no effect on Fas-induced apoptosis of immature osteoclasts. A partial abrogation of Fas-induced inhibition of osteoblast differentiation assessed by the number of AP-positive osteoblast colonies and expression of osteoblast differentiation genes was also observed when osteoblastogenic cultures were pretreated with estradiol before Fas ligation. This confirms that estrogen withdrawal may have a direct effect on the activity of the Fas/Fasl system in osteoblast lineage cells. A similar effect of estradiol on the expression of Fas was also described in $\mathrm{T}$ lymphocytes as a mechanism of stimulation of thymocyte apoptosis and increased sensitivity to autoimmunity. ${ }^{35}$ On the other hand, estradiol had no effect on the expression of Fas in osteoclasts, which points to the conclusion that a decrease in the osteoclastogenic response in Fas-deficient mice is not driven by a direct influence of Fas/Fasl system on the osteoclast lifespan. Estradiol pretreatment even increased the number of dead cells in mature osteoclastogenic cultures, which is not surprising as apoptosis of mature osteoclasts has already been described as a direct effect of estradiol. ${ }^{36}$

Taken together, our results show the important role of Fas/ Fasl in in vivo regulation of bone mass by estrogens. Our results showed that estrogen supports Fas-mediated osteoclast apoptosis and counteracts the negative effect of Fas on osteoblast differentiation and survival in vivo. We also showed that estrogen withdrawal requires an active Fas receptor for the development of osteoporotic phenotype, and that this effect involves mainly osteoblast lineage cells. Further analysis of the molecules involved in the regulatory effect of Fas on osteoblast-lineage cell survival and differentiation is needed to better define specific targets for the treatment of osteoresorptive disorders. 


\section{ACKNOWLEDGEMENTS}

RANKL was a kind gift from Amgen Inc. We thank Professor Dr Markus M. Simon for providing us the Fas -/- mice. We thank Mrs. Katerina ZrinskiPetrovic for her technical assistance. This work was supported by the grant from the Welcome Trust (CRIGS 073828/Z/03/Z) and the grants from the Croatian Ministry of Science, Education and Sports (108-1080229-0140, 108-1080229-0142, and 108-1080229-0341).

\section{DISCLOSURE/CONFLICT OF INTEREST}

The authors declare no conflict of interest.

1. Jilka RL, Hangoc G, Girasole G, et al. Increased osteoclast development after estrogen loss: mediation by interleukin-6. Science 1992;257: 88-91.

2. Kousteni $S$, Chen JR, Bellido T, et al. Reversal of bone loss in mice by nongenotropic signaling of sex steroids. Science 2002;298:843-846.

3. Tomkinson A, Reeve J, Shaw RW, et al. The death of osteocytes via apoptosis accompanies estrogen withdrawal in human bone. J Clin Endocrinol Metab 1997;82:3128-3135.

4. Denault JB, Salvesen GS. Caspases: keys in the ignition of cell death Chem Rev 2002;102:4489-4500.

5. Hehlgans T, Pfeffer K. The intriguing biology of the tumour necrosis factor/tumour necrosis factor receptor superfamily: players, rules and the games. Immunology 2005;115:1-20.

6. Siegel RM, Chan FK, Chun HJ, et al. The multifaceted role of Fas signaling in immune cell homeostasis and autoimmunity. Nat Immuno 2000;1:469-474.

7. Ishimaru N, Saegusa K, Yanagi K, et al. Estrogen deficiency accelerates autoimmune exocrinopathy in murine Sjogren's syndrome through fas-mediated apoptosis. Am J Pathol 1999;155:173-181.

8. Jaita G, Candolfi M, Zaldivar V, et al. Estrogens up-regulate the Fas/FasL apoptotic pathway in lactotropes. Endocrinology 2005;146:4737-4744.

9. Suzuki $A$, Enari $M$, Eguchi $Y$, et al. Involvement of Fas in regression of vaginal epithelia after ovariectomy and during an estrous cycle. EMBO J 1996;15:211-215.

10. Garcia-Moreno C, Catalan MP, Ortiz A, et al. Modulation of survival in osteoblasts from postmenopausal women. Bone 2004;35:170-177.

11. Kovacic N, Lukic IK, Grcevic D, et al. The Fas/Fas ligand system inhibits differentiation of murine osteoblasts but has a limited role in osteoblast and osteoclast apoptosis. J Immunol 2007:178:3379-3389.

12. Adachi $M$, Suematsu $S$, Kondo $T$, et al. Targeted mutation in the Fas gene causes hyperplasia in peripheral lymphoid organs and liver. Nat Genet 1995;11:294-300

13. Katavic V, Lukic IK, Kovacic N, et al. Increased bone mass is a part of the generalized lymphoproliferative disorder phenotype in the mouse. J Immunol 2003;170:1540-1547.

14. Grcevic D, Lee SK, Marusic A, et al. Depletion of CD4 and CD8 T lymphocytes in mice in vivo enhances 1,25-dihydroxyvitamin D3stimulated osteoclast-like cell formation in vitro by a mechanism that is dependent on prostaglandin synthesis. J Immunol 2000;165: 4231-4238.

15. Stahlberg A, Hakansson J, Xian X, et al. Advanced quantitative realtime PCR in clinical diagnostics and CDNA microarray validation. 1st European Conference in Functional Genomics and Diseases. Prague, Czech Republic, 2003.

16. University of Connecticut Health Center. Genome anatomy project for the osteoprogenitor lineage. Available from: http:// skeletalbiology.uchc.edu/30_ResearchProgram/304_gap/index.htm..
17. Bu R, Borysenko CW, Li Y, et al. Expression and function of TNF-family proteins and receptors in human osteoblasts. Bone 2003;33:760-770.

18. Hatakeyama S, Tomichi N, Ohara-Nemoto Y, et al. The immunohistochemical localization of Fas and Fas ligand in jaw bone and tooth germ of human fetuses. Calcif Tissue Int 2000;66:330-337.

19. Ogawa $Y$, Ohtsuki $M$, Uzuki $M$, et al. Suppression of osteoclastogenesis in rheumatoid arthritis by induction of apoptosis in activated CD4+ T cells. Arthritis Rheum 2003;48:3350-3358.

20. Wu X, McKenna MA, Feng X, et al. Osteoclast apoptosis: the role of Fas in vivo and in vitro. Endocrinology 2003;144:5545-5555.

21. Nagata S, Golstein P. The Fas death factor. Science $1995 ; 267$ : 1449-1456.

22. Eischen $\mathrm{CM}$, Schilling JD, Lynch $\mathrm{DH}$, et al. Fc receptor-induced expression of Fas ligand on activated NK cells facilitates cell-mediated cytotoxicity and subsequent autocrine NK cell apoptosis. J Immunol 1996;156:2693-2699.

23. Kiener PA, Davis PM, Rankin BM, et al. Human monocytic cells contain high levels of intracellular Fas ligand: rapid release following cellular activation. J Immunol 1997;159:1594-1598.

24. De Maria R, Testa U, Luchetti L, et al. Apoptotic role of Fas/Fas ligand system in the regulation of erythropoiesis. Blood 1999;93: 796-803.

25. Krum SA, Miranda-Carboni GA, Hauschka PV, et al. Estrogen protects bone by inducing Fas ligand in osteoblasts to regulate osteoclast survival. EMBO J 2008;27:535-545.

26. Nakamura T, Imai Y, Matsumoto T, et al. Estrogen prevents bone loss via estrogen receptor alpha and induction of Fas ligand in osteoclasts. Cell 2007;130:811-823.

27. Kameda $\mathrm{T}$, Mano $\mathrm{H}$, Yuasa $\mathrm{T}$, et al. Estrogen inhibits bone resorption by directly inducing apoptosis of the bone-resorbing osteoclasts. J Exp Med 1997;186:489-495.

28. Cenci S, Weitzmann MN, Roggia C, et al. Estrogen deficiency induces bone loss by enhancing T-cell production of TNF-alpha. J Clin Invest 2000;106:1229-1237.

29. Park H, Jung YK, Park OJ, et al. Interaction of Fas ligand and Fas expressed on osteoclast precursors increases osteoclastogenesis. J Immunol 2005;175:7193-7201.

30. Jilka RL, Takahashi K, Munshi M, et al. Loss of estrogen upregulates osteoblastogenesis in the murine bone marrow. Evidence for autonomy from factors released during bone resorption. J Clin Invest 1998;101:1942-1950.

31. Kawakami A, Eguchi K, Matsuoka N, et al. Fas and Fas ligand interaction is necessary for human osteoblast apoptosis. J Bone Miner Res 1997;12:1637-1646.

32. Tsuboi M, Kawakami A, Nakashima T, et al. Tumor necrosis factor-alpha and interleukin-1beta increase the Fas-mediated apoptosis of human osteoblasts. J Lab Clin Med 1999;134:222-231.

33. Kuo PL. Myricetin inhibits the induction of anti-Fas IgM-, tumor necrosis factor-alpha- and interleukin-1beta-mediated apoptosis by Fas pathway inhibition in human osteoblastic cell line MG-63. Life Sci 2005;77:2964-2976.

34. Kuo PL, Huang YT, Chang $\mathrm{CH}$, et al. Fraxetin inhibits the induction of anti-Fas IgM, tumor necrosis factor-alpha and interleukin-1 betamediated apoptosis by Fas pathway inhibition in human osteoblastic cell line MG-63. Int Immunopharmacol 2006;6:1167-1175.

35. Do $Y$, Ryu $S$, Nagarkatti $M$, et al. Role of death receptor pathway in estradiol-induced T-cell apoptosis in vivo. Toxicol Sci 2002; 70:63-72.

36. Syed F, Khosla S. Mechanisms of sex steroid effects on bone. Biochem Biophys Res Commun 2005;328:688-696. 